\title{
Lung ultrasound in the diagnosis and management of acute viral bronchiolitis; a systematic review
}

\author{
Christos Kogias ${ }^{1}$, Spyridon Prountzos ${ }^{1}$, Efthymia Alexopoulou ${ }^{1}$, and Konstantinos Douros ${ }^{1}$ \\ ${ }^{1}$ National and Kapodistrian University of Athens Faculty of Medicine
}

October 31, 2021

\begin{abstract}
Objective: Acute viral bronchiolitis (AVB) is one of the most common viral infections and the most common lower respiratory tract infection in the first year of life. Current guidelines recommend that medical history and physical examination have the main role in the diagnosis of AVB. Lung ultrasound (LUS) has not been included in the diagnostic algorithm so far. The aim of this systematic review is to collect all available studies concerning the role of LUS in the diagnosis and management of AVB. Methods: PubMed - MEDLINE, Scopus and ScienceDirect databases were searched for trials reporting on LUS examination in the diagnosis and management of AVB in paediatric patients. Results: A total of seventeen studies matching our eligibility criteria were analyzed for the purposes of this review and their results were categorized into six major fields each one of them answering to a question. There are several LUS scores that evaluate the severity of sonographic findings in children with AVB. The findings on LUS and chest radiography are comparable and LUS has a significant role in further management of AVB in the Paediatric Emergency Department. LUS score is correlated to the clinical course of AVB and it can predict both the duration of hospitalization and the need for respiratory support. Conclusion: Current literature supports that LUS could have comparable efficiency with chest radiography concerning the diagnosis of AVB and it could predict the length of hospital stay and the need of oxygen supply.
\end{abstract}

Lung ultrasound in the diagnosis and management of acute viral bronchiolitis; a systematic review

Christos Kogias $\mathrm{MD}^{1}$, Spyridon Prountzos $\mathrm{MD}^{2}$, Efthymia Alexopoulou MD, $\mathrm{PhD}^{3}$, Konstantinos Douros $\mathrm{MD}, \mathrm{PhD}^{4}$

${ }^{1}$ MSc candidate, National and Kapodistrian University of Athens, Faculty of Medicine, Athens, Greece

$2 \mathrm{PhD}$ candidate, $2^{\text {nd }}$ Department of Radiology, University General Hospital "Attikon", National and Kapodistrian University of Athens, Faculty of Medicine, Athens, Greece

${ }^{3}$ Professor of Paediatric Radiology, $2^{\text {nd }}$ Department of Radiology, University General Hospital "Attikon", National and Kapodistrian University of Athens, Faculty of Medicine, Athens, Greece

${ }^{4}$ Paediatric Pulmonologist and Allergist, Associate Professor of Paediatrics, $3^{\text {rd }}$ Department of Paediatrics, University General Hospital "Attikon", National and Kapodistrian University of Athens, Faculty of Medicine, Athens, Greece

Correspondence:

Christos Kogias, MD, National and Kapodistrian University of Athens, Faculty of Medicine, University General Hospital "Attikon", Rimini 1, Chaidari 12462, Athens, Greece

Email: christoskogias.ped@gmail.com 
Conflicts of interests: The authors declare that there is no conflict of interests.

Funding: This research received no external funding.

\begin{abstract}
Objective: Acute viral bronchiolitis (AVB) is one of the most common viral infections and the most common lower respiratory tract infection in the first year of life. Current guidelines recommend that medical history and physical examination have the main role in the diagnosis of AVB. Lung ultrasound (LUS) has not been included in the diagnostic algorithm so far. The aim of this systematic review is to collect all available studies concerning the role of LUS in the diagnosis and management of AVB. Methods: PubMed - MEDLINE, Scopus and ScienceDirect databases were searched for trials reporting on LUS examination in the diagnosis and management of AVB in paediatric patients. Results: A total of seventeen studies matching our eligibility criteria were analyzed for the purposes of this review and their results were categorized into six major fields each one of them answering to a question. There are several LUS scores that evaluate the severity of sonographic findings in children with AVB. The findings on LUS and chest radiography are comparable and LUS has a significant role in further management of AVB in the Paediatric Emergency Department. LUS score is correlated to the clinical course of AVB and it can predict both the duration of hospitalization and the need for respiratory support. Conclusion: Current literature supports that LUS could have comparable efficiency with chest radiography concerning the diagnosis of AVB and it could predict the length of hospital stay and the need of oxygen supply.
\end{abstract}

Keywords: Lung [Mesh]; Ultrasound [Mesh]; Ultrasonography [Mesh]; Bronchiolitis [Mesh]; Bronchiolitis, Viral [Mesh]; Infants [Mesh]; Radiography [Mesh]

\title{
Introduction
}

Acute viral bronchiolitis (AVB) is a clinical syndrome with varying symptomatology depending on the viral type. The two main viruses causing AVB are respiratory syncytial virus (RSV) and rhinovirus, with the former being more crucial for infants. In developed countries, AVB is the most common reason for hospitalization in the first twelve months of life ${ }^{1,2}$. Up to $6 \%$ of the patients with AVB require hospitalization to the paediatric intensive care unit $(\mathrm{PICU})^{3}$. Seasonal epidemics are usually observed during late autumn and early spring ${ }^{4,5}$.

AVB is characterized by extensive inflammation and oedema of the tracheobronchial tree, along with increased mucus production and apoptosis of airway epithelial cells ${ }^{6}$. Its clinical presentation begins with mild non-specific symptoms such as cough, runny nose, and low-grade fever followed by a gradual exacerbation of the symptoms leading to the development of respiratory distress. Lung auscultation reveals widespread fine end-inspiratory crackles, wheezing and prolonged expiration ${ }^{6,7}$.

The diagnosis of AVB up until now is based on medical history and physical examination. The disease lacks a pathognomonic radiological pattern ${ }^{8,9}$. The sensitivity of chest radiography has been estimated between 20 and $96 \%{ }^{10}$. Chest radiography can be helpful in severe cases. In typical cases, it does not add significantly to the diagnostic process ${ }^{11}$. Nevertheless, it is performed in about $50 \%$ of admitted infants with $\mathrm{AVB}^{12-14}$, mainly to rule out bacterial pneumonia, and results in significant exposure to ionizing radiation ${ }^{15}$ and unnecessary antibiotic prescription ${ }^{16}$. Most guidelines suggest oxygen therapy along with hydration and nutrition ${ }^{17}$.

Lung ultrasound (LUS) has been proposed as a diagnostic tool for many neonatal and paediatric conditions affecting the lung parenchyma such as transient tachypnea of the newborn ${ }^{18}$, respiratory distress syndrome ${ }^{19}$, and pneumonia ${ }^{20}$. Due to its rapid, repeatable, and non-ionizing nature, it is of great value in paediatric patients and is considered a useful alternative for examining the lungs.

During LUS, sound waves move slowly through the air which is the main component of normal lung parenchyma ${ }^{21}$. Thus, in the normally aerated lung, the pleura is the only visible structure since air has a high-acoustic impedance preventing the visualization of the parenchyma. Beyond the pleural line, in the 
healthy lung, artefacts are visualized representing reverberating waves which are produced by bouncing off the echo between the pleural line and probe. These stationary lines that are rather regularly spaced and arrayed parallel to the pleura, are called A-lines ${ }^{22}$. B-lines represent discrete, ray-like, vertical hyperechoic reverberation artefacts that arise from the pleural line and extend to the bottom of the screen, moving synchronously with the sliding lung 22,23 .

Currently, LUS is not considered a widespread tool in the diagnosis and clinical management of bronchiolitis. In the following text, we review the current evidence on the potential usefulness of LUS in AVB.

\section{Method}

Systematic literature research was performed in three different databases, MEDLINE, Scopus and ScienceDirect. The search included the keywords "bronchiolitis", "acute bronchiolitis", "acute viral bronchiolitis", "lung ultrasound", "lung ultrasonography" and the boolean operators "AND" and "OR". The initial search yielded 39 articles in MEDLINE, 110 in Scopus and 99 in ScienceDirect databases that were scanned for relevance. Ultimately, seventeen studies were found to be eligible and relative to our topic and were included in the present systematic review. All eligible papers have been published in the last ten years, starting from 2011.

This review has been conducted according to the Preferred Reporting Items for Systematic Review and Meta-Analyses (PRISMA) Guidelines ${ }^{24}$.

\section{Results}

Seventeen studies were eligible for use in this systematic review, which reported on LUS performed in infants with AVB and its role in the diagnosis and management of this pathological entity (Table 1). We sorted these studies into groups, according to their objective for further stratification of the results in our review.

Is there a feasible scoring system for stratifying AVB severity when performing LUS?

Four studies attempted to characterize and score LUS findings in patients with acute bronchiolitis. BuenoCampaña et al's ${ }^{31}$ scoring system is a multivariate system consisting of LUS score performed on the third day of respiratory distress along with clinical and epidemiological information in order to develop a predictive tool that addresses the risk for any type of respiratory support in infants with the diagnosis of AVB. One-hundred forty-five (145) infants (median age of 1.7 months, $47.6 \%$ females) diagnosed with moderate bronchiolitis [Wood-Down-Ferres Score (WDFS) ${ }^{43}$ 4, Interquartile Range (IQR) 4-6] were enrolled in the study. Isolated posterior consolidations showed the highest sensitivity [74.6\%, Confidence Interval (CI) 95\%: 61.6 - 85\%], followed by confluent B-lines in the posterior area (72.9\%) and $>3$ B-lines in the anterior area (74.6\%). LUS score showed a moderate prognostic accuracy, with an Area Under the Curve (AUC) of 0.84 (CI 95\%: 0.781 - 0.909). A cut-off point of 3.5 in the LUS showed good sensitivity and acceptable specificity. Clinical score WDFS [?] 6 on admission had a sensitivity of $56.1 \%$ and specificity of $85.9 \%$ for the need for respiratory support. Combining clinical predictors (age $<1$ month and WDFS [?] 6 points) with LUS findings in the new score, sensitivity improved to $89.1 \%$, though specificity decreased to $56 \%$ in comparison with these same clinical predictors used separately. High sensitivity allows using the score as a screening tool, with a good Negative Predictive Value (NPV) of $88.7 \%$, and a low proportion of false negatives of $10.9 \%$. Basile et $\mathrm{al}^{40}$ managed to correlate clinical and LUS scores between one-hundred six (106) infants (median age of 71 days) diagnosed with bronchiolitis and twenty-five (25) infants of the control group while evaluating the concordance of the two sonographers taking part in the study. The main LUS findings were B-lines, subpleural lung consolidations, bilateral involvement of intercostal spaces, and high involvement of posterior and paravertebral areas. Interobserver agreement on the severity of bronchiolitis was high (agreement: 90.6\%, expected agreement: $52.3 \%$, Cohen's kappa statistic $(\mathrm{K})=0.8$, Standard error $=0.0765, \mathrm{z}=10.19, \mathrm{p}=$ 0.000), along with high interobserver concordance on the basis of the ultrasound findings (Cohen's kappa coefficient: agreement $=89.6 \%$, expected agreement $46.4 \%, \mathrm{~K}=0.8$, Standard error $=0.07, \mathrm{z}=11.33, \mathrm{p}=$ 0.000). LUS permitted the identification of all of the twenty-nine (29) infants who received oxygen therapy, sharing common findings in the LUS such as $>6$ intercostal spaces involved and subcentimeter consolidations in the posterior and paravertebral area with a specificity of $98.7 \%$ (95\% CI: 93\% - 99.8\%), a sensitivity of 
96.6\% (95\% CI: $82.2 \%$ - 99.4\%), a Positive Predicted Value (PPV) of $96.6 \%$ (95\% CI: $82.2 \%$ - 99.4\%) and NPV of $98.7 \%$ (95\% CI: $92.95 \%$ to $99.8 \%$ ). Varshney et al ${ }^{39}$ characterized LUS findings in children [?] 2 years of age presenting to the Paediatric Emergency Department (PED) with signs of respiratory tract infection and wheeze. Also, they evaluated the diagnostic accuracy of these findings among infants with different diagnoses and the interobserver correlation between an expert and a novice sonologist. LUS was positive in $42 \%(39 / 94)$ of patients and findings included multiple B-lines in $80 \%(31 / 39)$, consolidations in $64 \%(25 / 39)$ among which $10 \%(4 / 39)$ were large consolidations, total pleural line abnormalities $10 \%(4 / 39)$ and total pleural fluid $18 \%$ (7/39). When categorized by final diagnosis, a positive LUS was found in $46 \%(33 / 72)$ of patients with bronchiolitis, $0 \%(0 / 14)$ of patients with asthma, 100\% (4/4) of patients with pneumonia, and in $50 \%(2 / 4)$ of those with concomitant asthma and pneumonia. This study indicated good reliability between the novice and expert sonologist [K=0.68 (95\% CI: $0.54-0.82)]$ for a positive LUS ([?] 1 positive finding). Almost perfect agreement was found between raters for each finding: $\mathrm{K}=0.88$ (95\% CI: $0.78-0.98$ ) for [?] 3 B-lines per intercostal space, 0.62 (95\% CI: 0.42 - 0.82) for small consolidations, 0.88 (95\% CI: 0.66 - 1.00) for large consolidations and 0.55 (95\% CI: 0.26 - 0.84) for pleural abnormalities. Ingelse et $\mathrm{al}^{26}$ tried to semi-quantify loss of aeration and to associate serial LUS score with oxygenation anomaly (as a marker of disease severity) in children receiving invasive mechanical ventilation (IMV) for severe bronchiolitis. There was a positive association between both LUS scores and Oxygen Saturation Index (OSI) as our primary outcome marker for disease severity on the first day of invasive mechanical ventilation, albeit the degree of correlation was modest, but no positive association after the first day of invasive mechanical ventilation.

In conclusion, scoring systems evaluating B-lines, consolidations, and the number of intercostal spaces involved seem to be the most efficient at predicting disease severity in children with AVB. Subpleural lung parenchyma, along with posterior and paravertebral areas tend to associate with higher disease likelihood. Also, these scoring systems tend to show a good interobserver agreement and concordance when performed by radiologists and paediatricians.

Are LUS and chest radiography findings comparable?

The first study that compared the diagnostic accuracy of LUS and chest X-ray (CXR) in children with the diagnosis of bronchiolitis was published by Caiulo et $\mathrm{al}^{41}$. Fifty-two (52) patients (28 males, 2.1 months median age) who underwent a study with chest radiography took part in this study. Control group included fifty-two (52) patients admitted for gastroesophageal reflux (median age of 3 months, 28 males). LUS findings in the AVB group revealed subpleural lung consolidation, numerous compact B-lines and pleural line abnormalities in all infants with $\mathrm{p}<0.001$ in comparison with the control group. It revealed subtle findings such as a minimal pleural effusion in 3/52 infants and small pneumothorax in one infant. Chest radiography study was less sensitive revealing lung consolidation in 16/52 infants, peribronchial wall thickening in 21/52 infants and lung hyperexpansion of the chest in one infant, while it was reported as normal in 14/52 infants with bronchiolitis. Nine (9) patients whose clinical score was consistent with the diagnosis of bronchiolitis had a negative chest radiography, though a positive LUS. The authors managed to correlate clinical and LUS findings as having an almost linear relationship. Interpretation time greatly varied between LUS which had no delays, while chest radiography had an average of 4 hours and 45 minutes delay since its request. La Regina et $\mathrm{al}^{27}$ assessed a ninety-two-infant cohort (29 of them being the control group), described lung ultrasound findings and compared lung ultrasound and chest radiography findings in infants hospitalized with bronchiolitis. A significant correlation was found both on the presence of subpleural lung consolidations in LUS and chest radiography $(\mathrm{K}=82 \%)$ and on abnormalities of the pleural line in LUS and air trapping in chest radiography $(\mathrm{K}=75 \%)$. Later, Biagi et $\mathrm{al}^{32}$ investigated the diagnostic accuracy and reliability of LUS for the detection of pneumonia in hospitalized children with AVB and evaluated the agreement between LUS and CXR in diagnosing pneumonia in these infants. A total of eighty-seven (87) patients (mean age of 5.7 months) were enrolled in the study. A total of 25/87 patients with AVB were diagnosed with concomitant pneumonia. Of these twenty-five (25) patients, all but one was positive for parenchymal consolidation consistent with pneumonia in chest radiography, while all patients were positive for pneumonia according to LUS findings. Chest radiography set a false-positive diagnosis in eight (8) infants. LUS set a false-positive diagnosis for pneumonia in ten (10) cases, all but one consisting of subcentimetric consolidation 
pneumonia. The remaining patient had a false-positive diagnosis with a consolidation $>1 \mathrm{~cm}$ on LUS. Chest radiography had only one false-negative case while LUS had none. Overall, chest radiography showed a sensitivity of $96 \%$ (95\% CI: $88.8 \%-98.8 \%$ ) and specificity of $87.1 \%$ (95\% CI: $77.8 \%-93.0 \%$ ) in identifying children with AVB affected by a concomitant bacterial pneumonia, while LUS had a sensitivity of $100 \%$ (95\% CI: $94.7 \%-99.9 \%)$ and a specificity of $83.9 \%$ (95\% CI: $74.1 \%-90.6 \%)$. A strong correlation between chest radiography and LUS in diagnosing bacterial pneumonia [Spearman's rank correlation coefficient (rho or rs) $=0.638, \mathrm{p}<0.0001$ ] was shown using Spearman's rho test. When only consolidation size $>1 \mathrm{~cm}$ was considered positive for pneumonia, the correlation in the study grew further $(\mathrm{rs}=0.68)$. A perfect agreement in diagnosing bacterial pneumonia by LUS $(\mathrm{K}=0.93)$ was shown by Cohen's kappa between paediatrician and radiologist sonologists in the first 30 patients. A less strong agreement $(\mathrm{K}=0.74)$ was shown between the two expert radiologists that interpreted the positive CXRs in these infants. The final point this study suggests is that a positive LUS with consolidations sized $>1 \mathrm{~cm}$ may avoid the need to perform chest radiography in these patients. Kağan Özkaya et al ${ }^{32}$ study included seventy-six (76) infants (median age of 6 months) of which seventy-four (97\%) infants had a positive LUS, while only forty-two (55.3\%) had a positive chest radiography. Consequently, a statistically significant difference between chest radiography and LUS was shown in children with AVB (McNemar test $\mathrm{p}<0.001)$ indicating that LUS has a better value as a diagnostic tool. Jaszczolt et al's ${ }^{34}$ study further confirmed that transthoracic lung ultrasound can be a reliable tool in the diagnosis of lung lesions caused by RSV infection. This study compared LUS and chest radiography findings in twenty-six (26) children (median age of 7 months) with clinical diagnosis of AVB requiring hospital admittance. An abnormal chest radiography was found in 4/26 infants, consisting of peribronchial cuffing and hilar enlargement. On the other hand, 21/26 infants had abnormal findings in the LUS, consisting of inflammatory consolidations $>1 \mathrm{~cm}$ in $11 / 26$ patients, alveolar-interstitial syndromes in $11 / 26$ patients, small consolidations $<1 \mathrm{~cm}$ in $8 / 26$ patients, interstitial syndrome in $6 / 26$ patients and a small amount of pleural effusion in $3 / 26$ patients. Ultimately, the study showed that LUS had a higher sensitivity in detecting small amounts of pleural effusion (0 in CXR vs 3 in LUS) or lesions within the pulmonary parenchyma (a total of 11 large and 8 small consolidations in LUS vs 0 infiltrations in CXR) compared to chest radiography.

To summarize, all studies concluded that LUS tends to be more sensitive in detecting large and subtle parenchymal findings and extrapulmonary sequelae in children with AVB compared to chest radiography. Furthermore, LUS serves as a good prognostic tool in the context of predicting the clinical course of patients and is quicker in interpretation than chest radiography.

What is the role of the LUS in the PED?

Six studies attempted to evaluate the usefulness of the LUS in the PED and four of them qualified it as important. Zoido Garrote et $\mathrm{al}^{37}$ found a correlation between the LUS score and the need of PICU admission [Odds Ratio $(\mathrm{OR})=2.5$ (95\% CI: $1.1-5.9), \mathrm{p}=0.035$ ]. Bobillo - Perez et $\mathrm{al}^{25}$, in their study, concluded that LUS score could predict the PICU admission more accurately than clinical score (AUC 0.932 (95\% CI: $0.873-0.990)$ vs. $0.675(95 \%$ CI: $0.556-0.794)$, respectively). Supino et $\mathrm{al}^{30}$ conducted a study consisting of 76 infants (median age of 90 days) in which LUS was performed in the PED by emergency paediatricians. The study concluded that there was a significant difference in LUS scores between the infants who needed advanced respiratory support [High Flow Nasal Canula (HFNC) or Helmet Continuous Positive Airway Pressure (HCPAP)] on admission and those who did not $(\mathrm{rs}=0.35, \mathrm{p}=0.003)$. This study claims that LUS score can predict the need for oxygen support in infants with AVB evaluated in the PED and further strengthen the concept of LUS in the PED to better define the prognosis as a first assessment tool. In the study of San Sebastian Ruiz et $\mathrm{al}^{29}$ the relevance between LUS findings in admission at the PED and the need of respiratory support in infants with the diagnosis of AVB was assessed. Two-hundred (200) infants (median age of 5 months) were included in the study which concluded that abnormal ultrasound patterns tend to be associated with higher rates of admission and need of oxygen therapy. Among infants needing respiratory support the vast majority $(86,9 \%)$ had an abnormal LUS pattern upon arrival at the PED. Interstitial pattern along with consolidation tends to be the main predictor of worse outcome. 
However, two studies showed no benefit in the use of LUS in the PED. Cohen et al ${ }^{36}$ study aimed to describe the prevalence of B-lines in children younger than 2 years old presenting to the PED with wheezing, determined the interrater reliability of LUS findings in this setting and evaluated the association of B-lines with atopy and other clinical findings. Twenty-nine (29) infants (mean age of 291 days) were included. Of them, twenty-one (21) infants (72\%) had [?] 2 compact B-lines in LUS and no subpleural consolidations or pleural effusion. The presence of compact B-lines was strongly associated with older age $(p=0.001)$ and was negatively associated with atopy $(\mathrm{p}=0.0001)$. Major issue was the poor interrater reliability was proved in interpretation of the LUS examination $(\mathrm{K}=0.08)$. So, compact B-lines were associated with a lack of atopy, suggesting that compact B-lines may be associated with viral-induced wheezing rather than atopic wheezing. The previously described statistical pattern seems to follow Varshney et al's ${ }^{39}$ study. They described LUS findings in children [?] 2 years of age, presenting to the PED with signs of a respiratory tract infection and wheezing, while evaluating both the diagnostic accuracy of these findings among infants with different diagnoses and the interobserver correlation between an expert and a novice sonologist. LUS was positive in $42 \%(39 / 94)$ of the patients with findings of multiple B-lines in $80 \%(31 / 39)$ of them, consolidation in $64 \%(25 / 39)$, total pleural line abnormalities in $10 \%(4 / 39)$ and total pleural fluid in $18 \%(7 / 39)$. When categorized by final diagnosis, a positive LUS was found in $46 \%(33 / 72)$ of patients with bronchiolitis, $0 \%$ $(0 / 14)$ of patients with asthma, $100 \%(4 / 4)$ of patients with pneumonia and in $50 \%(2 / 4)$ of those with concomitant asthma and pneumonia. Furthermore, this study indicated good reliability between the novice and expert sonologist $[\mathrm{K}=0.68$ (95\% CI: $0.54-0.82)]$ for a positive LUS ([?] 1 positive findings) and an almost perfect agreement was found between raters for each finding.

Summarizing the facts stated above, we conclude that LUS can be performed safely in the setting of PED both by expert sonologists and novice users with good agreement in the findings. A higher LUS score in the PED can predict a worse outcome when patients need hospital admittance and also correlates to the need of advanced respiratory support in the form of HFNC or HCPAP of inpatients.

Is there a correlation between LUS score and clinical course?

Caiulo et $\mathrm{a}^{41}$ evaluated the concordance between LUS and clinical findings. They found that there is a parallel correlation between clinical and sonographic findings in all infants, between the clinical improvement or deterioration and the decrease or the increase in the number of consolidations, respectively. A significant correlation between LUS and clinical score $(\mathrm{rs}=0.62, \mathrm{p}<0.001)$ has also been stated by La Regina et $\mathrm{al}^{27}$ who described LUS findings in cases and controls and compared LUS and chest radiography findings in hospitalized infants. In the same way, Basile et $\mathrm{al}^{40}$ found agreement between clinical and sonographical evaluation of the attending physician and the sonographer, respectively (agreement: $90.6 \%$, expected agreement: $52.3 \%, \mathrm{~K}=0.8$, Std error $=0.0765, \mathrm{z}=10.19, \mathrm{p}=0.000)$. Supino et $\mathrm{al}^{30}$ compared and evaluated for the first time the severity of AVB clinically and sonographically in seventy-six (76) previously healthy infants (median age of 90 days), during their first assessment carried out by emergency paediatricians in the PED. Infants who needed HFNC or HCPAP had a more severe sonographic $(\mathrm{p}=0.028)$ and clinical score $(\mathrm{p}=0.004)$, compared to those who did not need it. Also, LUS and clinical scores were able to predict the need for oxygen support in the PED. Zoido Garrote et al's ${ }^{37}$ observational, prospective study evaluated the correlation between LUS findings in an early stage and the clinical progression in fifty-nine (59) infants diagnosed with mild-to-moderate bronchiolitis. Pulmonary involvement was estimated on the basis of an LUS score and WDF and Saint Joan de Deu Hospital (HSJD) clinical scores. The median LUS score was 6 points in patients who did not acquire admission compared to 9 in patients admitted to the hospital, with 17 of the patients requiring transfer to the PICU $(\mathrm{p}<0.001)$. The LUS score correlated moderately to the WDFS ( rho $=0.504, \mathrm{p}<0.001)$ and the HSJD score $($ rho $=0.518, \mathrm{p}<0.001$ ). Moustafa Abdel Kader et $\mathrm{al}^{38}$ assessed the role of LUS in the evaluation of infants with AVB, a study that included twenty-five (25) infants with suspected bronchiolitis. A decrease in size or disappearance of the subpleural hypoechoic areas in the LUS, thus an improvement in the LUS score, was correlated with clinical improvement. Ahmet Kağan Özkaya et $a^{32}$ demonstrated a significant correlation between the modified Bronchiolitis Severity clinical Score (mBSS) and the LUS score in children with AVB $(\mathrm{r}=0.698, \mathrm{p}<0.001)$. LUS score showed a better performance in predicting hospital admission with AUC of $0.804(95 \%$ CI: $0.71-0.89, \mathrm{p}<0.001)$ in 
comparison with AUC for the clinical score that was $0.814(95 \% \mathrm{CI}: 0.71-0.91, \mathrm{p}<0.001)$

Conversely, some studies failed to demonstrate a correlation between LUS score and the clinical course of AVB. Di Mauro et al ${ }^{28}$ tried to evaluate the relationship between an ultrasound score and the clinical progression of bronchiolitis: the need for supplemental oxygen, duration of oxygen therapy, and length of hospital stay. Of eighty-three (83) patients who took part in the study, the LUS score was weakly correlated with both the clinical score at admission $[\mathrm{rho}=0.23(95 \% \mathrm{CI}: 0.02-0.43), \mathrm{p}=0.036]$ and the clinical score at discharge $[\mathrm{rho}=0.30(95 \% \mathrm{CI}: 0.07-0.51), \mathrm{p}=0.001]$. Similarly, Taveira et al ${ }^{35}$ found no statistically significant correlation between LUS and WDF scores on admission for 47 infants (median age of 32 days) with severe AVB ( $\mathrm{p}=0.57)$.

Although several of the studies included in this review successfully showed a good correlation between sonographic scores and the clinical course of the disease, either in the improvement or in the deterioration, there is a dichotomy of data from other authors, which points to the nonuniformity of AVB as a disease, possibly due to the nature of its causative factors.

Can LUS score predict the length of hospital stay?

A number of studies managed to determine the correlation between LUS score and hospital stay. La Regina et $\mathrm{al}^{27}$ found a correlation between LUS score and the length of hospitalization ( $\mathrm{rho}=0.42, \mathrm{p}<0.001$ ), as well as, Bueno - Campaña et $\mathrm{al}^{31}$ (rho $=0.401, \mathrm{p}<0.0001$ ). Di Mauro et $\mathrm{al}^{28}$ concluded that the mean length of hospitalization was $4.6 \pm 2.0$ days and that the length of hospital stays correlates with LUS score $\left[\mathrm{rs}=0.3(95 \% \mathrm{CI}: 0.1-0.5)\right.$, standard error $\left.=0.09, \mathrm{t}=3.7, \mathrm{p}<0.0001, \mathrm{r}^{2}=0.13\right]$. This study validates a previously published ultrasound score for bronchiolitis by Basile et $\mathrm{al}^{40}$ and underlines the correlation between LUS findings and the duration of hospital stay. Zoido-Garrote et al ${ }^{37}$ evaluated the correlation between LUS findings in an early stage and the clinical progression. LUS score was associated with long hospital stay. More specifically, a correlation was proved between each 5-point increase in the LUS score and increase in length of stay by 1.2 days $(95 \%$ CI: $0.55-1.86, \mathrm{p}<0.001)$. Interobserver agreement between the two trainee paediatricians and the expert paediatrician who conducted the LUS was high 0.917 (95\% CI: 0.854-0.956). Bobillo-Perez et $\mathrm{al}^{25}$ found a greater correlation between LUS score and the length of hospital stay $($ rho $=0.764)$ in comparison with clinical score $(\mathrm{rho}=0.477)$. Nevertheless, the study of Taveira et al ${ }^{35}$ found no such correlation $(\mathrm{p}=0.38)$.

Overall, a great number of studies confirmed that LUS scores correlate to the duration of hospitalization. Higher LUS scores are associated with longer hospital stays.

Can LUS score predict the need for oxygen supply?

Ingelse et $\mathrm{al}^{26}$ described LUS abnormalities in 17 children receiving IMV in the PICU. Two different LUS scores were assessed, counting B-line score and aeration score. A positive, moderate correlation was found between both LUS scores and OSI on the first day of IMV ( $\mathrm{rs}=0.52, \mathrm{p}=0.034$ for LUS counting B-line score and $\mathrm{rs}=0.57, \mathrm{p}=0.017$ for LUS aeration score). There was found no correlation between LUS scores and cumulative fluid balance and no difference in LUS scores among patients with or without bacterial coinfection ( $\mathrm{p}=0.068$ for counting B-line score and $\mathrm{p}=0.248$ for LUS aeration score). Moreover, LUS scores did not correlate with the duration of IMV $(\mathrm{p}=0.938$ for counting B-line score and $\mathrm{p}=0.894$ for LUS aeration score). Interobserver agreement between observers 1 and 2 who performed separately the LUS was moderate [Intraclass Correlation Coefficient (ICC) was 0.731 for counting B-line score and 0.697 for LUS aeration score). A higher agreement was found between the third observer who assessed the video clips and observer $1\left(\mathrm{ICC}=0.890\right.$ for counting B-line score and 0.785 for LUS aeration score). Basile et al ${ }^{40}$ assessed 29 infants with AVB who required supplementary oxygen. LUS permits the identification of those infants that are in need of supplementary oxygen with a specificity of $98.7 \%$ (95\% CI: $93 \%-99.8 \%$ ), a sensitivity of $96.6 \%$ (95\% CI: $82.2 \%-99.4 \%$ ), a PPV of $96.6 \%$ (95\% CI: $82.2 \%-99.4 \%$ ) and a NPV of $98.7 \%$ (95\% CI: $92.95 \%$ - 99.8\%). Thus, LUS findings correlate with the clinical evaluations in infants with AVB and can identify those infants in need of oxygen supplementation with high specificity. Supino et al ${ }^{30}$ found no correlation between LUS score and oxygen saturation upon admission in PED. Nonetheless, LUS score and the duration 
of oxygen therapy during admission were found to correlate evidently ( $\mathrm{rs}=0.35, \mathrm{p}=0.003$ ). Infants who received HCPAP ventilation had a LUS score of 2 (1-4) and a clinical score of 7 (5-9) in comparison with infants who did not need respiratory support, with scores of 1 (1-3) and $5(2-6)$, respectively $(\mathrm{p}=0.003$ and $\mathrm{p}=0.004$, respectively). Consequently, both clinical and ultrasound scores can predict the need for oxygen support in infants with AVB evaluated in the PED. Di Mauro et $\mathrm{al}^{28}$ evaluated the relationship between an ultrasound score and the need and duration for oxygen therapy. The LUS score was $4.5+-1.7$ (range: 2.0 8.0) in patients that required supplemental oxygen, different from the one of the not supplemented infants $(2.5+-1.8$, range: $0.0-6.0, \mathrm{p}<0.001)$. While the need for supplemental oxygen seemed to be associated with the LUS score [OR $=2.2(95 \%$ CI: $1.5-3.3)$ standard error $=0.45, \mathrm{z}=3.9, \mathrm{p}<0.0001]$, the duration of oxygen supplementation was not associated $[\mathrm{rs}=0.01(95 \% \mathrm{CI}$ : $-0.01-0.03)$, standard error $=0.01, \mathrm{t}=$ $\left.0.9, \mathrm{p}=0.363, \mathrm{r}^{2}=0.03\right]$. In the study of Zoido Garrote et $\mathrm{al}^{37}$ a significant correlation was proved between each 5-point increase in the LUS score and duration of oxygen therapy by 0.87 days (95\% CI: $0.26-1.48, \mathrm{p}=$ 0.006). Moreover, San Sebastian Ruiz et $\mathrm{al}^{29}$ estimated that among infants in need of $\mathrm{O}_{2} /$ High Flow Oxygen (HFO) at admission, $86.7 \%$ had an abnormal LUS and among the ones in need of NIV at admission, $87.5 \%$ had an abnormal LUS. The corresponding rates related to respiratory support at 48 hours after admission are $14(77.8 \%)$ and $14(87.5 \%)$, respectively. Only three infants $(3.5 \%)$ with normal LUS patterns needed respiratory support at admission and six $(7 \%)$ at 48 hours. In concordance with the previous studies, La Regina et $\mathrm{al}^{27}$ found that the need for oxygen therapy was more frequent in the patients with higher LUS scores $(\mathrm{p}<0.001)$. Bueno-Campana et $\mathrm{al}^{31}$ found that certain LUS findings correlate significantly with the need for respiratory support. More than 3 anterior, bilateral B-lines had Relative Risk (RR) of 3.2 (95\% CI: $1.3-7.9, \mathrm{p}=0.009)$, anterior, bilateral, confluent B-lines had RR of $4.5(95 \%$ CI: $1.9-10.9, \mathrm{p}=0.001)$, anterior consolidation $>1 \mathrm{~cm}$ had RR of $2.4(95 \% \mathrm{CI}: 1.1-5.4, \mathrm{p}=0.034)$ and posterior consolidation $>1 \mathrm{~cm}$ had RR of 4.4 (95\% CI: $1.8-10.8, \mathrm{p}=0.001)$. Bobillo-Perez et $\mathrm{al}^{25}$ found a positive correlation between LUS score and the duration of any respiratory support $(\mathrm{rho}=0.763)$ in comparison with clinical score (rho $=0.462)$. La Regina et $\mathrm{al}^{27}$ noted a more frequent need of oxygen supplementation in infants with AVB

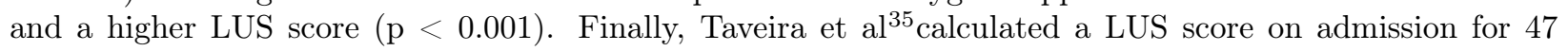
infants with the diagnosis of severe bronchiolitis. The LUS score on admission $(3.5+-2.6)$ did not correlate significantly with the length of NIV $(69+-68.6$ hours, rho $=0.1, \mathrm{p}=0.51)$ or the length of oxygen therapy $(3+-3.4$ days, rho $=0.26, \mathrm{p}=0.08)$. Though, a significant correlation was noted between the number of affected intercostal spaces on the right and the length of oxygen supply ( rho $=0.318, \mathrm{p}=0.037)$.

\section{Discussion}

AVB is the most common LRTI in the first year of life and current guidelines indicate that its diagnosis is based solely on medical history and clinical examination. Chest radiography is a valuable and widely available method for the diagnosis of a multitude of lung diseases however its use should be limited, especially in children, because of the ionizing radiation exposure.

In recent years, lung ultrasonography has been established as a useful tool in pulmonology and lately, it has been introduced into paediatric pulmonology. It is a diagnostic modality with excellent attributes since it is non-radiating, with reproducible results that can be used bedside even by non-radiologists. San Sebastian Ruiz et $\mathrm{al}^{29}$, Supino et $\mathrm{al}^{30}$, Ingelse et $\mathrm{al}^{26}$ and Zoido-Garrote et $\mathrm{al}^{37}$ found very good agreement in ultrasound findings between amateur and expert paediatricians and concluded that LUS is a bedside examination that can be very useful to paediatricians that are properly trained. Also, a study by Nadimpalli et $\mathrm{al}^{42}$ (not included in this review), evaluated the concordance in ultrasound findings among 6 trainee General Practitioners (GP). The GPs, after attending a 12-hour theoretical and practical session, conducted and interpreted a lung ultrasound examination in 60 children with the diagnosis of AVB or other viral LRTI. The inter-rater agreement between the GPs and the experts was good (Cohen's K: 0.81, 95\% CI: $0.74-0.87)$. The study demonstrated that non-radiologists physicians can be trained to effectively use lung ultrasonography for the diagnosis of pulmonary diseases in children.

Lung ultrasonography is a diagnostic tool that can predict the need for respiratory support and hospital admission. All ten studies ${ }^{25-31,35,37,40}$ found a significant correlation between lung ultrasound score and 
the need for oxygen supply and also that LUS score can help identify patients who will require respiratory support. Three studies assessed the correlation between LUS score and the duration of respiratory support. Two of them found a significant correlation ${ }^{30,37}$ while one study found no correlation between them ${ }^{28}$. It seems that combining lung ultrasonography findings with oxygen saturation measurements can help predict the need for oxygen supplementation. Another firm conclusion is that lung ultrasonography can predict the length of hospital stay in patients with AVB. $\mathrm{All}^{25,27,28,37}$ but one $\mathrm{e}^{35}$ of the studies that examined this issue found a significant correlation between the severity of LUS score with the number of additional in-hospital days.

Not all of the studies support that LUS and clinical scores correlate significantly. However, this lack of correlation may reside, at least in part, to the fact that clinical scores have moderate sensitivity and specificity and thus they cannot predict accurately the severity of the disease ${ }^{45}$. Even so, four studies support that LUS score can predict more accurately than the clinical score the need for PICU admission in patients with $\mathrm{AVB}^{25,29,30,37}$.

LUS was compared to chest radiography regarding the sensitivity and specificity in detecting pathological findings compatible with AVB. Four out of five studies that examined this question estimated that LUS was superior to chest radiography in sensitivity though it lagged behind in specificity ${ }^{32-34,41}$. Chest radiography is the preferred method when there is diagnostic uncertainty due to its higher specificity. Currently, guidelines recommend medical history and clinical examination as diagnostic tools in AVB $^{44}$. However, lung ultrasound with its good sensitivity and the absence of ionizing radiation can have a significant complementary role, in the primary assessment and follow-up of AVP. Given all the promising aforementioned attributes of LUS it would be reasonable to consider it as a first-line examination in children with AVB in the PED, in the near future. Although the existence of multiple lung ultrasound scoring systems can be confusing for sonographic users of different backgrounds and levels of experience, the same basic principles are applied each time, allowing for a steep learning curve when performing LUS, increasing its reproducibility and, ultimately, making it a realistic goal as a first-line examination in AVB.

In conclusion, lung ultrasound is considered a simple, safe, quick, non-invasive, low-cost, real-time examination that allows physicians to evaluate AVB patients in PED and during their stay in hospital and change the management of the disease according to sonographic findings. Future prospective studies are needed to shed further light on the validity of LUS and its proper place in the management of children with AVB.

\section{Hosted file}

Kogias_References.docx available at https://authorea.com/users/443806/articles/543715-lungultrasound-in-the-diagnosis-and-management-of-acute-viral-bronchiolitis-a-systematicreview

\section{Hosted file}

Kogias_Flow_diagram.docx available at https://authorea.com/users/443806/articles/543715-lungultrasound-in-the-diagnosis-and-management-of-acute-viral-bronchiolitis-a-systematicreview

\section{Hosted file}

Kogias_Table.docx available at https://authorea.com/users/443806/articles/543715-lungultrasound-in-the-diagnosis-and-management-of-acute-viral-bronchiolitis-a-systematicreview 